\title{
Low-temperature Fourier transform infrared spectra and hydrogen bonding in polycrystalline L-alanine
}

\author{
M. Rozenberg ${ }^{\mathrm{a}, *}$, G. Shoham ${ }^{\mathrm{a}}$, I. Reva ${ }^{\mathrm{b}}$, R. Fausto ${ }^{\mathrm{b}}$ \\ ${ }^{a}$ Department of Inorganic and Analytical Chemistry, The Hebrew University of Jerusalem, Givat Ram, Jerusalem 91904, Israel \\ ${ }^{\mathrm{b}}$ Department of Chemistry, University of Coimbra, Coimbra 3004-535, Portugal
}

Received 4 February 2003; accepted 25 February 2003

\begin{abstract}
The 400-4000 $\mathrm{cm}^{-1}$ FTIR spectra of pure NH and isotopically substituted (10 and 90\% doped ND/NH) polycrystalline $\mathrm{L}$-alanine were recorded in the temperature range $10-300 \mathrm{~K}$. The observed temperature dependence and isotopic shifts behavior enabled to identify, in the spectra of the doped crystals, three well-separated bands ascribable to either the NH or ND stretching vibrations associated with the three different types of hydrogen bonds existing in the crystal. The observed red shifts of these bands relative to the frequency of a reference "free" NH (or ND) stretching mode were found to correlate well with the H-bond distances found in the crystal and provide an indirect way of estimating the enthalpies associated with each type of H-bond found in the crystal. In the low-frequency deformation and torsional spectral region (below $2000 \mathrm{~cm}^{-1}$ ), several bands, which were found to be affected by isotopic substitution, were identified as belonging to the $\mathrm{NH}_{3}^{+}$group. Several bands show splitting at low temperatures, indicating the occurrence of a significant reorganization in the crystal structure, which with all probability results mainly from changes in the proton positions. Finally, the literature assignments of the IR spectra of both crystalline $\mathrm{NH}_{3}^{+}$and $\mathrm{ND}_{3}^{+}$L-alanine were revised taking into consideration their temperature dependence and behavior upon deuteration.
\end{abstract}

(C) 2003 Elsevier B.V. All rights reserved.

Keywords: L-Alanine crystal; H-bonding; Low-temperature FTIR

\section{Introduction}

The properties of proton vibrational modes of crystalline L-alanine have never been correlated with $\mathrm{H}$-bond energies and distances, despite L-

\footnotetext{
* Corresponding author. Tel.: +972-2-658-5177; fax: +9722-658-5319.

E-mail address: markroz@chem.ch.huji.ac.il

(M.
}

alanine has been previously the subject of several spectroscopic (IR and Raman) studies in both mono- and polycrystalline forms at room and low temperatures ([1 and references cited therein, 24]). The obvious reason for this is that, as it is usually observed for crystals with differently $\mathrm{H}$ bonded multiple $\mathrm{OH}$ - or $\mathrm{NH}$ - groups, in crystalline L-alanine the proton stretching mode $\left(v_{1}\right)$ gives rise to a broad and extensively structured band, thus preventing any reliable direct identification of 
the features due to individual $\mathrm{NH}$ bonds by conventional methods [5]. In addition, the outof-plane bending mode $\left(v_{4}\right)$, which is very sensitive to H-bonding [6-9], can also be expected to be a sensitive probe of this interaction in amino acids, since the bands due to this mode (found below $1000 \mathrm{~cm}^{-1}$ ) can result from a $\mathrm{H}$-bound state of wagging and torsional vibrations of the $\mathrm{NH}_{3}^{+}$ group. However, no studies have been reported until now describing an approach based on this vibration, in particular for crystalline L-alanine.

Nevertheless, structural data unambiguously show that three different types of $\mathrm{H}$-bonds exist in the crystal of L-alanine [10,11], and these should give rise to different $\mathrm{NH}$ stretching frequencies. The first attempt to identify in the IR spectra of crystalline L-alanine bands which can be assigned to the structurally sensitive vibrational modes of individual protons and to correlate them with both $\mathrm{H}$-bond distances and energies is made in this paper.

The approach used here is based on two main special experimental techniques, which were used concomitantly: the use of isotopically doped crystals and the application of very low working temperatures.

As it will be described in detail below, the use of doped crystals enabled the identification of the IR bands due to isotopically labeled ND-alanine molecules in a NH-alanine crystal, and of NHalanine molecules in a ND-alanine crystal. But this technique has not only the advantage of providing a reliable and direct way to unequivocally identify the spectroscopic features due to the NH (or ND) vibrational modes. At doping concentrations less than $10 \%$, the minority of $\mathrm{D}$ - or $\mathrm{H}$-containing molecules is surrounded by an H- or D-bulk, respectively. Under these circumstances, their vibrational modes are free from both internal dynamic coupling, because of mass and/or symmetry changes, or coupling with neighboring $\mathrm{H}$ bonds. Then, if the isotopically substituted atom can assume three non-equivalent structural positions, three separate bands are expected in the IR spectrum.

The simultaneous application of low temperature considerably enhances the power of using isotopic doping to study H-bonding in crystals, since under these experimental conditions thermal broadening, which results from interaction of the higher frequency modes of the $\mathrm{H}$-bonded protons (such as $v_{1}$ and $v_{4}$ ) with the low-frequency deformational modes of the hydrogen bond, is minimized [12-14]. Thus, the intrinsic resolution of the IR bands considerably improves upon lowering the temperature, the average bandwidths decreasing from ca. 200 to $10 \mathrm{~cm}^{-1}$ in both $v_{1}$ and $v_{4}$ spectral ranges when going from room temperature to $20 \mathrm{~K}$.

It is also important to mention that, to the best of our knowledge, no reliable empirical correlations between spectroscopic parameters related either with $v_{1}$ or $v_{4} \mathrm{NH}$ vibrational modes and crystal-state $\mathrm{H}$-bond energies $[6,15]$ were reported until now for nitrogen-containing systems with relevant biological interest. This contrasts with the situation found for hydroxyl-substituted species, such as polyols and pyranosides [5,16], for which such kind of correlations, involving the $\mathrm{OH}$ stretching and out-of-plane bending modes, could already been established. All these facts constitute additional elements stressing the interest and opportunity of the present study.

\section{Experimental}

The FTIR spectra of polycrystalline pure NH-, $2-5 \%$ ND- and 90-95\% ND-L-alanine in a $\mathrm{KBr}$ (1:200) disc, attached to the cold finger of an APD Cryogenics closed-cycle helium refrigeration system with a DE-202A expander, were recorded with a Mattson Infinity 60AR series FTIR spectrometer, with spectral resolution of $1 \mathrm{~cm}^{-1}$. The temperature $(10-300 \mathrm{~K})$ was measured directly at the sample holder by a silicon diode temperature sensor connected to the Digital Temperature Indicator model SI 9650 (Advanced Research Systems, Inc.) with a stability of $\pm 0.2 \mathrm{~K}$. The temperature-induced spectral changes observed for all substances were found to be reversible and highly reproducible. Deuterated samples were obtained from commercial L-alanine (Sigma) by recrystallization from $\mathrm{D}_{2} \mathrm{O}$ (Aldrich). 


\section{Results and discussion}

\subsection{The range $4000-2000 \mathrm{~cm}^{-1}$}

The temperature dependence of the spectra of pure $(100 \% \mathrm{H})$ and isotopically doped (NH-Lalanine in a D-bulk and ND-L-alanine in a $\mathrm{H}$ bulk) samples of L-alanine is shown in Fig. 1. The spectra now obtained resemble closely those recently measured at $10 \mathrm{~K}$ [4]. As expected, the components of the $v_{1}$ bulk band become narrower upon cooling and the band structure becomes more pronounced because of the strengthening of the resonant interactions with the NH (or ND) bending overtones or combination modes - the frequency of these latter increases while that of $v_{1}$
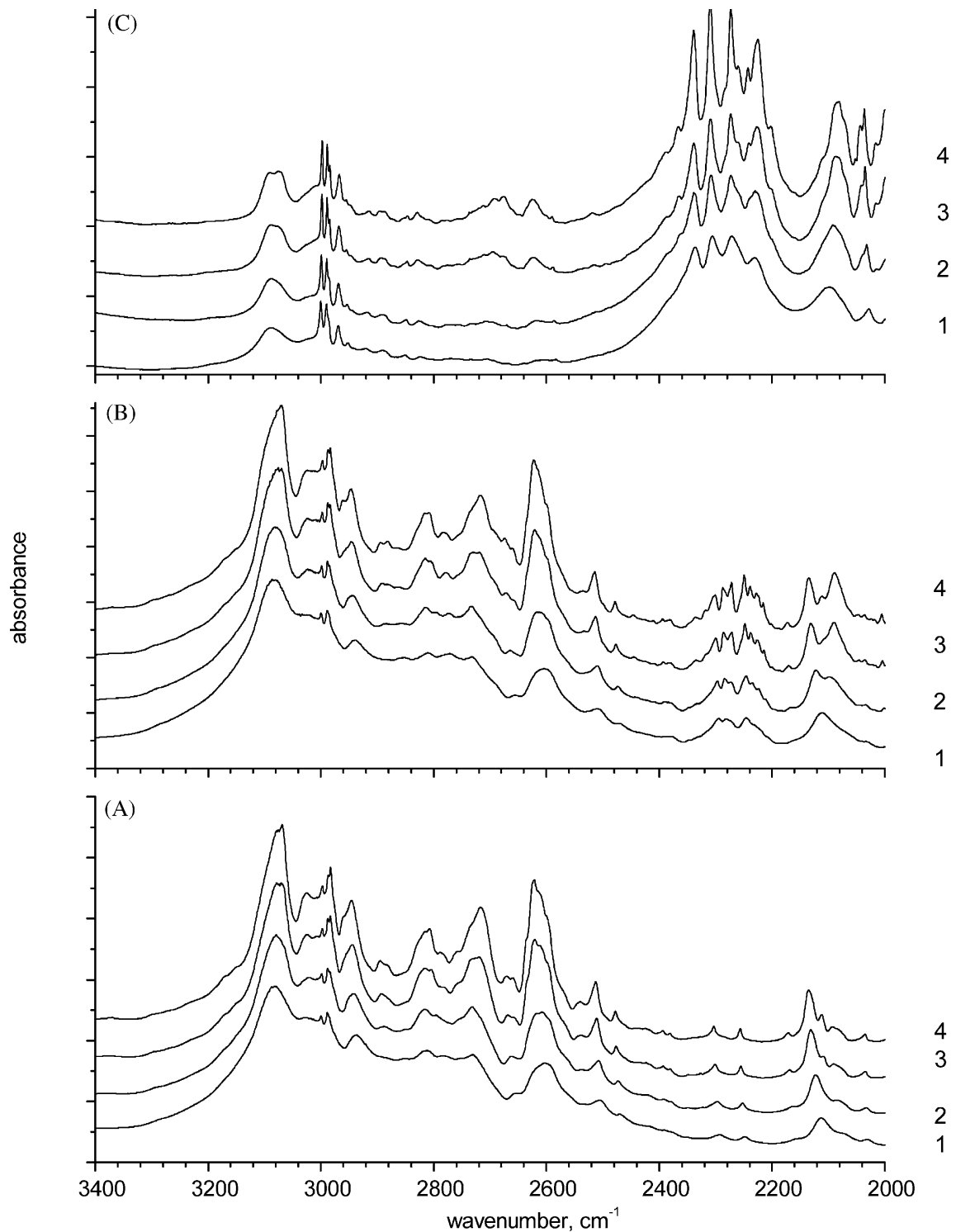

Fig. 1. Spectra of L-alanine, containing $100 \% \mathrm{NH}(\mathrm{A}),>90 \% \mathrm{NH}(\mathrm{B})$ and $<10 \% \mathrm{H}(\mathrm{C})$ at temperatures $300 \mathrm{~K}(1), 200 \mathrm{~K}(2), 100 \mathrm{~K}$ (3) and $10 \mathrm{~K}(4)$ in the range $3400-2000 \mathrm{~cm}^{-1}$. 
decreases with the strengthening of the H-bond due to the cooling. From Fig. 1, it can be seen that the bands due to the minor isotopic species can only be clearly observed at low temperatures. The spectra of all three samples, recorded at $10 \mathrm{~K}$, are correlated with each other in Fig. 2. The observed frequencies and bandwidths are given in Table 1 together with the proposed assignments.

In the NH stretching region, the spectrum of the doped $\mathrm{NHD}_{2}^{+}$molecules (Fig. 1C) consists of three bands, with peak maxima at 3076, 2990 and $2680 \mathrm{~cm}^{-1}$, corresponding to the three possible positions of the $\mathrm{H}$ atom in the molecule. At $100 \mathrm{~K}$, the first band splits into two components, separated by $22 \mathrm{~cm}^{-1}$ (Figs. 1 and 3A) with intensity ratio of $1: 1$. The second band is partially masked by the intense $\mathrm{CH}$ stretching bands (which are clearly identifiable since they exhibit a characteristic narrow band profile), but at $10 \mathrm{~K}$ it can be clearly observed. The third band shows a strong resonant interaction with the first overtone of the $\mathrm{NH}$ bending mode, which fundamental is observed at ca. $1370 \mathrm{~cm}^{-1}$ (this band disappears upon deuteration, confirming its origin).

In the ND stretching region, the spectrum of the $\mathrm{NDH}_{2}^{+}$molecules (in the $\mathrm{NH}$ bulk crystal) closely resembles the $\mathrm{NH}$ stretching region of the $\mathrm{NHD}_{2}^{+}$ species in the ND bulk crystal discussed above.

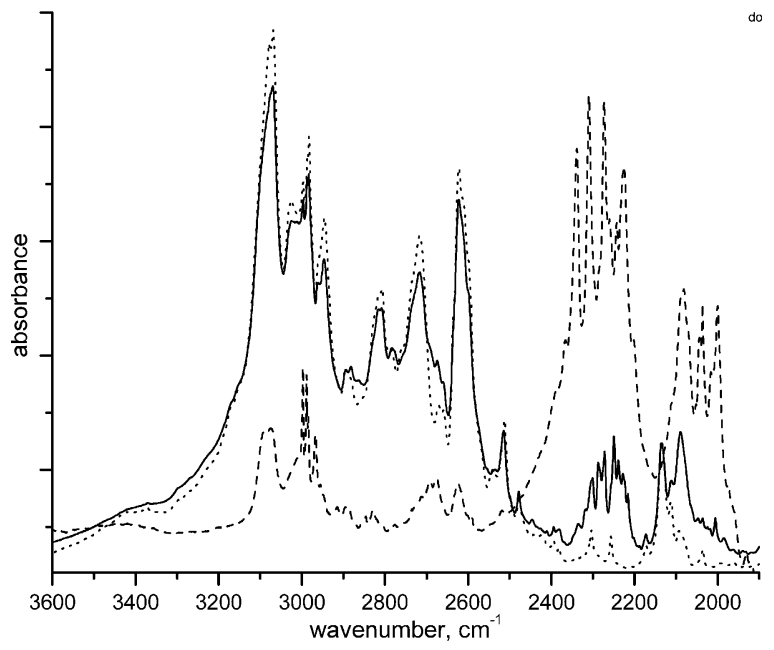

Fig. 2. Spectra of L-alanine, containing $100 \% \mathrm{NH}$ (dotted line), $>90 \% \mathrm{NH}$ (solid line) and $<10 \% \mathrm{H}$ (dashed line) at $10 \mathrm{~K}$ in the range $3600-1900 \mathrm{~cm}^{-1}$.
The two highest frequency bands observed in this spectrum (2287 and $2240 \mathrm{~cm}^{-1}$; Fig. 1B) are relatively broad $\left(30 \mathrm{~cm}^{-1}\right)$ and disturbed due to resonant interactions, leading to the appearance of a band structure similar to that corresponding to the ND bulk band profile (see Fig. 2). The third band, appearing at $2088 \mathrm{~cm}^{-1}$, is narrower and does also clearly reveal its involvement in a resonant interaction with the first overtone of the ND-deformation vibration (fundamental at 1056 $\mathrm{cm}^{-1}$ ), in a similar way to the equivalent band at $2680 \mathrm{~cm}^{-1}$ observed for the $\mathrm{NHD}_{2}^{+}$species. These bands are associated with the three possible positions of the $\mathrm{D}$ atom in the molecule.

The isotopic ratios of the three isotopically decoupled pairs of NH/ND stretching bands (at $10 \mathrm{~K})$ are $1.345,1.343$ and 1.280 , respectively. Hence, the isotopic ratios correlate well with the relative position of the bands: the smaller the isotopic ratio, the lower is the frequency of the band, corresponding to a stronger H-bond. Together with the intensity ratio of the three bands (ca. 1:1:1), these observations agree with the suggested assignment of each one of these bands to the $v_{1}$ mode originated in one of the three individual $\mathrm{NH}$ or $\mathrm{ND}$ bonds in $\mathrm{NH}_{3}^{+}$or $\mathrm{ND}_{3}^{+}$ groups. These were found to be involved in three different H-bonds in the L-alanine crystal, with associated $\mathrm{N}-\mathrm{H}$ bond lengths of $0.1047,0.1031$ and $0.1031 \mathrm{~nm}$ [10] $(0.1082,0.1044,0.1018 \mathrm{~nm}$ [11]).

The analysis of the structured band of pure $\mathrm{NH}(\mathrm{ND})$ L-alanine crystal, especially at room temperature, cannot be done on the basis of such an easy interpretation, due to the above-mentioned complexity due to intermode and intermolecular couplings and thermal broadening.

From the results now discussed, the reassignment of the bands observed at 1370 and 1056 $\mathrm{cm}^{-1}$ as corresponding to the NH and ND bending modes, respectively, could also be unequivocally established. The $1370 \mathrm{~cm}^{-1}$ band was previously ascribed to a $\mathrm{CH}_{3}$ bending mode $[1,2,4]$, but its behavior upon isotopic substitution supports the alternative assignment now proposed.

The IR spectrum of monomeric L-alanine zwitterions isolated in a $\mathrm{KBr}$ matrix has been obtained previously using the novel dissolution-spraying- 
Table 1

Peak frequencies, widths (in parentheses) and relative intensities (qualitative) of bands in pure and isotopically diluted L-alanine polycrystals at $10 \mathrm{~K}$ in the $3400-2000 \mathrm{~cm}^{-1}$ range

\begin{tabular}{|c|c|c|c|c|}
\hline$v(100 \% \mathrm{H})$ & $v, v^{\prime}(>90 \% \mathrm{H})$ & Assignment & $v, v^{\prime}(<20 \% \mathrm{H})$ & Assignment \\
\hline $3068.0(40) \mathrm{s}$ & $3069.2(40) s$ & & $3076(50) \mathrm{m}$ & $v\left(\mathrm{NHD}_{2}\right)^{+}$decoupled \\
\hline $3024.3(10) v w$ & 3024.3 & & $2997.8(5) \mathrm{m}$ & $v_{\mathrm{a}}\left(\mathrm{CH}_{3}\right)$ \\
\hline $2996.8(10) v w$ & $2996.8 v w$ & & $2990(80) \mathrm{m}$ & $v\left(\mathrm{NHD}_{2}\right)^{+}$decoupled \\
\hline $2982.9(10) w$ & $2983.3 w$ & $v\left(\mathrm{NH}_{3}\right)^{+}$ & $2989.7(5) \mathrm{m}$ & $v_{\mathrm{a}}\left(\mathrm{CH}_{3}\right)$ \\
\hline $2944.8(20) w$ & 2946.2 & & $2968.2(8) \mathrm{w}$ & $v\left(\mathrm{C}^{\alpha} \mathrm{H}\right)$ \\
\hline $2893.2(20) v w$ & $2881.0 v w$ & Centered at $\approx 2800 \mathrm{~cm}^{-1}$ & & \\
\hline $2814.0(30) w$ & $2814.0(30)$ & & & \\
\hline $2715.8(30) m$ & $2715.3(30) m$ & & $2677(60) \mathrm{w}$ & $v\left(\mathrm{NHD}_{2}\right)^{+}$decoupled \\
\hline $2620.8(30) s$ & $2622.7(40) s$ & & $2625(30) \mathrm{w}$ & $2 \delta\left(\mathrm{NH}_{3}^{+}\right)$ \\
\hline $2512.3 v w$ & $2514.3 v w$ & & & \\
\hline $2477.1 v w$ & $2478 v w$ & & $2338.8(10) s$ & \\
\hline \multirow[t]{2}{*}{$2302.6 \mathrm{vw}$} & $2300.2(10) \mathrm{vw}$ & & $2309.8(10) \mathrm{s}$ & Centered at $\approx 2270 \mathrm{~cm}^{-1}$ \\
\hline & $2287.0(30) w$ & $v\left(\mathrm{NDH}_{2}\right)^{+}$decoupled & $2273.2(10) s$ & \\
\hline $2255.8 \mathrm{vw}$ & $2240.0(30) w$ & $v\left(\mathrm{NDH}_{2}\right)^{+}$decoupled & $2225.0(10) \mathrm{s}$ & \\
\hline \multirow[t]{4}{*}{$2134.3(20) \mathrm{m}$} & $2133.4(20) \mathrm{m}$ & $\left(\mathrm{NH}_{3}\right)_{\text {bent }}^{+}+\left(\mathrm{NH}_{3}\right)_{\text {tors. }}^{+}$ & - & $v\left(\mathrm{ND}_{3}\right)^{+}$ \\
\hline & $2088.1(20) w$ & $v\left(\mathrm{NDH}_{2}\right)^{+}$decoupled & $2081.8(50) s$ & \\
\hline & & & $2036.5(10) s$ & Centered at $\approx 2040 \mathrm{~cm}^{-1}$ \\
\hline & & & $1999.8(10) s$ & \\
\hline
\end{tabular}

Note: bold — bands of decoupled stretching NH and ND modes; italic - band structure of broad NH or ND stretching bands in monoisotopic species.

deposition (DSD) technique [17]. The $v_{\mathrm{a}}{ }^{\prime}, v_{\mathrm{a}}$ and $v_{\mathrm{s}}$ $\mathrm{NH}$ stretching vibrations of the "free" $\mathrm{NH}_{3}^{+}$were assigned to the bands observed at 3314,3249 and $3143 \mathrm{~cm}^{-1}$, respectively. Observation of wellseparated $\mathrm{NH}$ asymmetric stretching bands $\left(v_{\mathrm{a}}{ }^{\prime}\right.$ and $v_{\mathrm{a}}$ ) indicates that under this experimental conditions the $\mathrm{NH}_{3}^{+}$group of the L-alanine zwitterion must be interacting with the $\mathrm{KBr}$ matrix $\left(\mathrm{Br}^{-}\right)$. However, since the observed frequencies are $300-600 \mathrm{~cm}^{-1}$ higher than those found in the crystal, it can be concluded that the $\mathrm{NH}_{3}^{+} / \mathrm{Br}^{-}$ interactions in the $\mathrm{KBr}$ matrix are considerably weaker than the intermolecular H-bonding interactions that are present in the crystalline state. In any case, the presence of the $\mathrm{NH}_{3}^{+} / \mathrm{Br}^{-}$interactions in the L-alanine zwitterions isolated in the $\mathrm{KBr}$ matrix does not allow us to use the data obtained for this system as a measure for the frequency of a free $\mathrm{NH}$ stretching vibration in the L-alanine zwitterions. Hence, for our further applications we accepted as reference frequency of a free $\mathrm{NH}$ stretching mode the mean value of $v_{\mathrm{a}}$ and $v_{\mathrm{a}}{ }^{\prime}$ calculated in [18] for L-alanine neutral form, i.e. $3380 \mathrm{~cm}^{-1}$. Once a suitable reference frequency value for the free NH stretching vibration in L-alanine could be defined, the empirical correlation between the observed frequency shift $\left(\mathrm{cm}^{-1}\right)$ upon hydrogen bond formation and the H-bond length (nm) [19] can be applied to the studied molecular system. The general expression of such empirical correlation, $\Delta v=r^{-6.1}$, was derived taking into consideration data collected for a considerable number of $\mathrm{H}$-bonded systems where the H-donor groups are not formally charged [19]. L-Alanine zwitterion is then the first molecular system bearing a formally charged $\mathrm{H}$ donor group to which this correlation is applied. The observed red shifts for the three observed NH stretching bands relative to the reference free $\mathrm{NH}$ frequency are 280,360 and $640 \mathrm{~cm}^{-1}$ (the shifts were reduced by $10 \%$ to take into account the necessary correction due to temperature effects [5]). Using these values in the above described empirical relationship yields $\mathrm{O} \cdots \mathrm{HN}^{+}$distances of $0.189,0.181$ and $0.165 \mathrm{~nm}$, respectively. These values are in a reasonable agreement with the experimental data obtained from X-ray and neutron diffraction structural studies [10,11]. For 

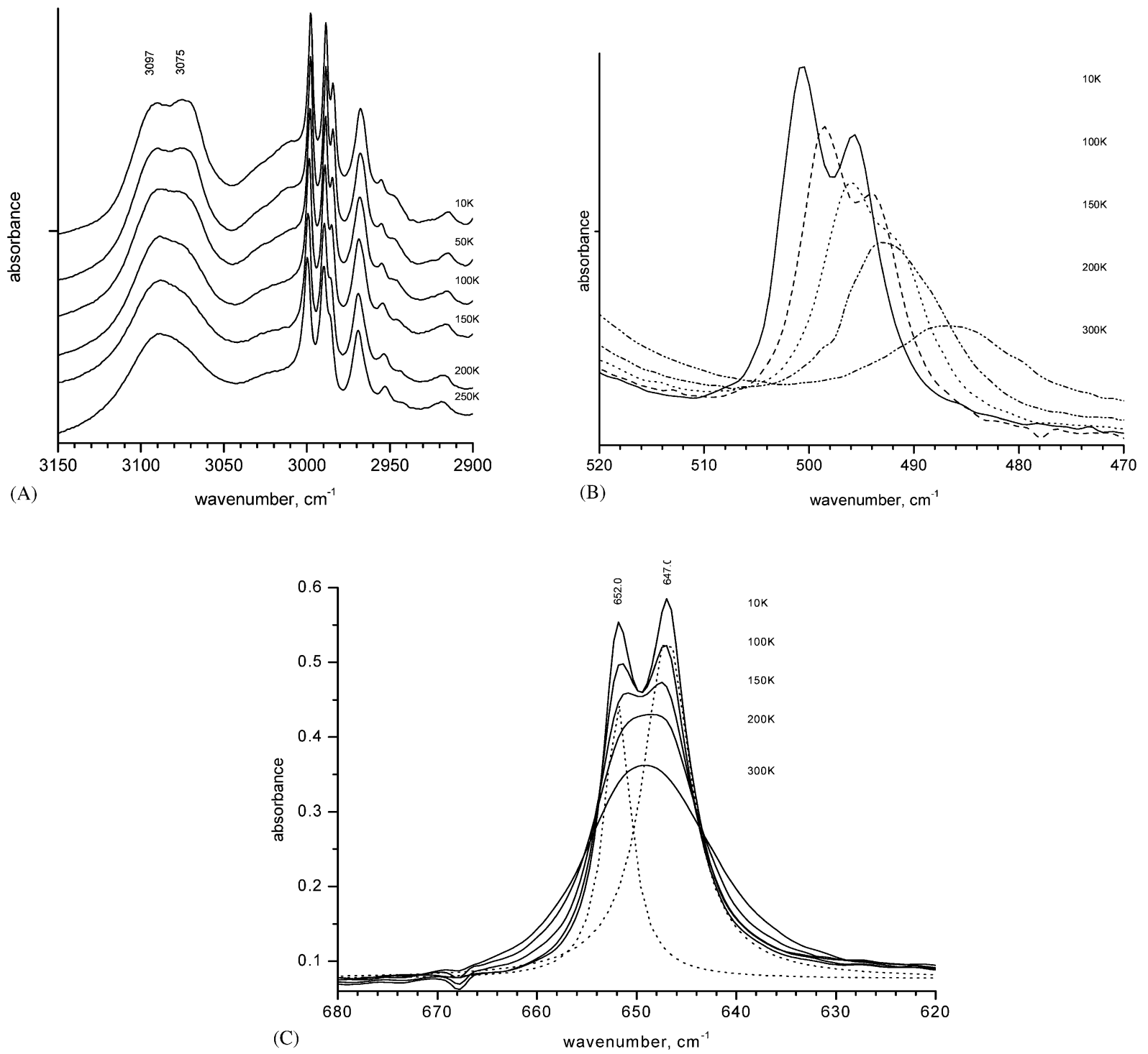

Fig. 3. Temperature changes of the bands: (A) $v_{1}(\mathrm{NH})$ in $>90 \% \mathrm{ND}$ L-alanine, (B) band at $500 \mathrm{~cm}^{-1}$ in pure $\mathrm{L}$-alanine and (C) band at $650 \mathrm{~cm}^{-1}$ in pure L-alanine (dotted line-deconvolution of the band at $10 \mathrm{~K}$ shows the component intensity ratio $1: 2$ ).

instance, the $\mathrm{H}$-bond distances obtained by neutron diffraction were $\mathrm{O}_{1} \cdots \mathrm{H}_{1}=0.186 \mathrm{~nm}$, $\mathrm{O}_{2} \cdots \mathrm{H}_{2}=0.183 \mathrm{~nm}$ and $\mathrm{O}_{2} \cdots \mathrm{H}_{3}=0.178 \mathrm{~nm}[10]$.

The IR spectra of the $\alpha$-modification of crystalline glycine doped with deuterium at low concentrations were previously measured at $77 \mathrm{~K}$ [20]. From the data shown in [20], the peak positions of the three bands corresponding to $v_{1}$ (ND) of the $\mathrm{NDH}_{2}^{+}$-glycine molecules were estimated as 2370 ,
2200 and $2080 \mathrm{~cm}^{-1}$. Assuming identical isotopic ratios for the three observed bands in glycine and L-alanine crystals, the position of the three $\mathrm{NH}$ decoupled bands in glycine were estimated as being 3170,2960 and $2660 \mathrm{~cm}^{-1}$, respectively. The corresponding red shifts of 190,380 and 650 $\mathrm{cm}^{-1}$ (with temperature correction) were calculated relatively to the reference frequency for the free $\mathrm{NH}$ vibration, which was here taken as being 
the same as for L-alanine. Hence, application of the empirical correlation [19] leads to estimations of the $\mathrm{H}$-bond distances in $\alpha$-crystalline glycine of $0.201,0.179$ and $0.164 \mathrm{~nm}$, which indeed are in fairly good agreement with neutron scattering data: $0.212,0.183$ and $0.173 \mathrm{~nm}$ [21].

Despite all the approximations made, the agreement obtained between the calculated and experimental $\mathrm{H}$-bond distances for both amino acids (glycine and L-alanine crystals) is good and demonstrates the applicability of the empirical correlation also to molecular systems bearing an $\mathrm{H}$-donor group formally charged. Note that for this kind of systems, hydrogen bonding may be expected not to be the main force determining the interatomic distances. In that case, the empirical relationship should not be applicable successfully. However, it does not seem to be the case for amino acids, indicating that, with all probability, the large volumes associated with the electron charge distributions in both the $\mathrm{NH}_{3}^{+}$and $\mathrm{CO}_{2}^{-}$groups reduce the importance of the electrostatic effects on the short-range order interaction and increase the influence of $\mathrm{H}$-bonding.

Another useful empirical relationship, which relates the frequency red shift of the stretching vibration of the $\mathrm{H}$-bond donor group due to the hydrogen bond formation $\left(\Delta v, \mathrm{~cm}^{-1}\right)$ with the square of the H-bonding enthalpy $(\Delta H, \mathrm{~kJ}$ $\left.\mathrm{mol}^{-1}\right),(\Delta H)^{2}=1.92(\Delta v-40)$, has been found for systems exhibiting shifts due to the H-bond formation from 100 to $850 \mathrm{~cm}^{-1}$ [15]. Though there is no possibility to check this correlation for amino acids with independent thermodynamic data, an approximate estimation can still be done. In the case of the L-alanine crystal, the Hbond energies associated with the three observed NH stretching bands $\left(3076,2990\right.$ and $\left.2680 \mathrm{~cm}^{-1}\right)$ resulting from this empirical correlation are 21.5, 25.0 and $34.0 \mathrm{~kJ} \mathrm{~mol}^{-1}$, respectively. The sum of all estimated $\mathrm{H}$-bond energies is then $80.5 \mathrm{~kJ}$ $\mathrm{mol}^{-1}$, which is close to the binding energy experimentally obtained for the charged alanine dimer, which amounts to $108 \mathrm{~kJ} \mathrm{~mol}^{-1}$ [22]. This comparison is reasonable since "the lowest energy structures of the glycine, alanine and glycinealanine proton-bound dimers indicate that the charge on the N-terminus of one amino acid is 'solvated' by both the carboxyl oxygen and the amine nitrogen of the other acid" [22], i.e. more than one $\mathrm{H}$-bond contribute to the dimer bonding energy.

\subsection{The $2000-400 \mathrm{~cm}^{-1}$ spectral region}

The spectra of crystalline L-alanine at different isotopic compositions and temperatures, in the region below $1300 \mathrm{~cm}^{-1}$, are shown in Fig. $4 \mathrm{~A}-\mathrm{C}$. Spectra of all three samples below $2000 \mathrm{~cm}^{-1}$ at 10 $\mathrm{K}$ are compared in Fig. 5. The bands appearing at ca. 1460, 1415, 921 and $770 \mathrm{~cm}^{-1}$ are observed in all these spectra, and thus are not affected by deuteration. Moreover, they do not change significantly with temperature. Hence, they should be assigned to skeletal or $\mathrm{CH}$ (either $\mathrm{CH}_{3}$ or $\mathrm{C}-\mathrm{H}$ ) bending modes (see Table 2).

In the spectrum of pure NH L-alanine (Fig. 4A), the bands at 972 and $500 \mathrm{~cm}^{-1}$ show prominent temperature changes - they develop on cooling, then increase their frequency and become progressively narrower. Below $200 \mathrm{~K}$, the band observed at $500 \mathrm{~cm}^{-1}$, as well as that appearing at 1160 $\mathrm{cm}^{-1}$, splits into two components (Fig. 3B). We shall discuss the peculiar temperature dependence exhibited by these bands later on. All these three bands are sensitive to deuteration, but the band at $972 \mathrm{~cm}^{-1}$ has no counterpart in the spectrum of ND L-alanine. The band observed at $650 \mathrm{~cm}^{-1}$ in the spectrum of pure $\mathrm{NH}$ L-alanine does not change position upon cooling but it splits into two components upon lowering the temperature (Fig. 3C). This band, like that appearing at 972 $\mathrm{cm}^{-1}$, is also sensitive to deuteration and has no counterpart in spectrum of ND L-alanine.

In the spectrum of doped $\mathrm{NDH}_{2}^{+}$molecules in the H-bulk (Fig. 4B), new low-intensity bands are observed, which belong to the isolated ND and $\mathrm{NH}_{2}$ deformational (torsion) modes of the $\mathrm{NDH}_{2}^{+}$ group. They can be clearly seen only in the spectra collected at a temperature below $100 \mathrm{~K}$ and experience a blue shift on cooling. We should note that some bands due to the ND-bonds are not seen in the range below $800 \mathrm{~cm}^{-1}$ because they are too weak within the experimental conditions used.

The frequencies and bandwidths of all the deuteration sensitive bands appearing in the spec- 


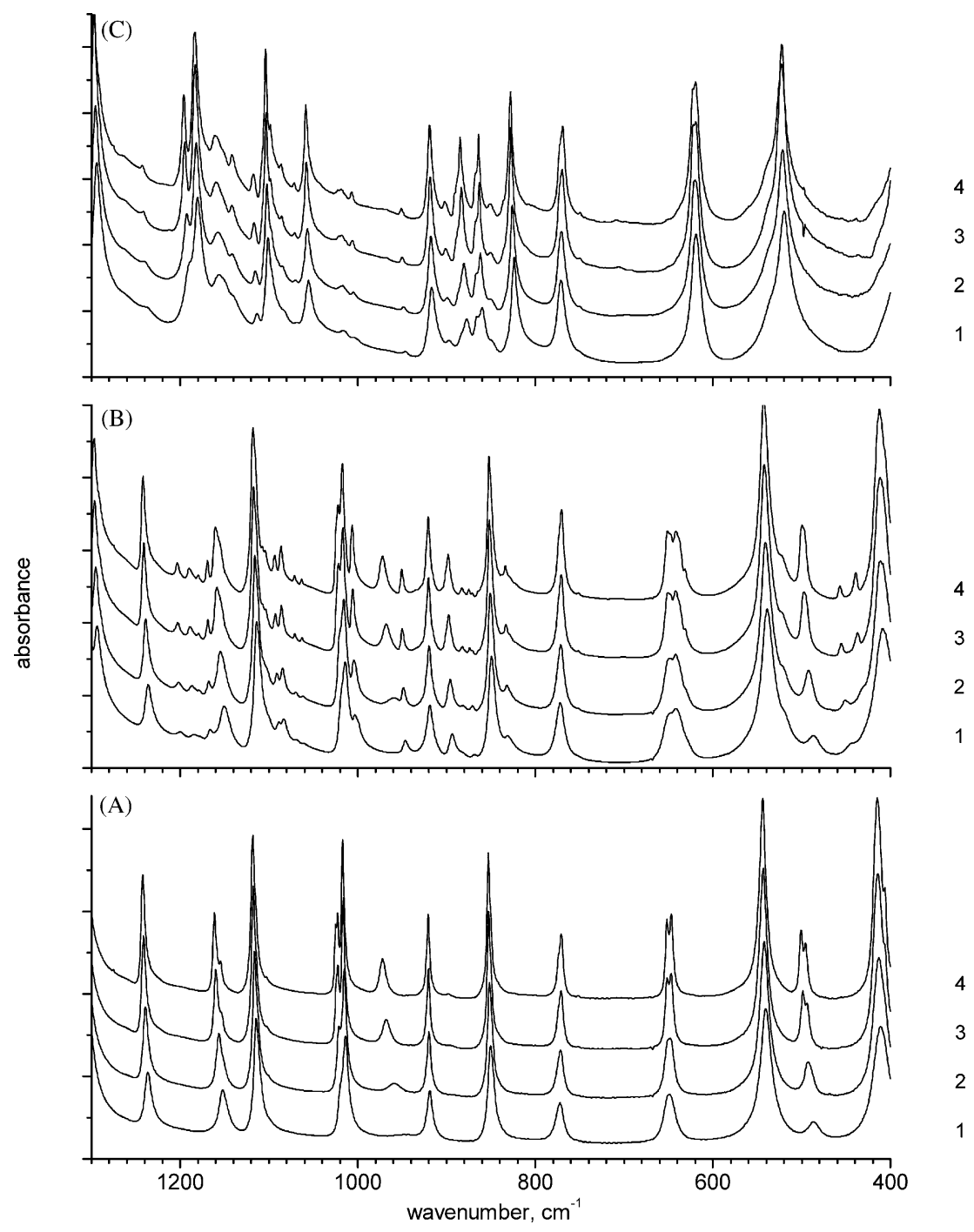

Fig. 4. Spectra of L-alanine, containing $100 \% \mathrm{NH}(\mathrm{A}),>90 \% \mathrm{NH}(\mathrm{B})$ and $<10 \% \mathrm{H}(\mathrm{C})$ at temperatures $300 \mathrm{~K}(1), 200 \mathrm{~K}(2), 100 \mathrm{~K}$ (3) and $10 \mathrm{~K}(4)$ in the range $1300-400 \mathrm{~cm}^{-1}$.

tral range below $2000 \mathrm{~cm}^{-1}$ are given in Table 2, for all three samples studied.

When the concentration of deuterium is close to $100 \%$ (Fig. 4C), some of the bands ascribed to the $\mathrm{NDH}_{2}^{+}$group disappear, while others increase their intensity. We suggest that the first set of bands belong to $\mathrm{NH}$ vibrations and the second to ND vibrations. At least eight bands could be assigned to NH vibrations. Some of these bands have their ND counterpart also observable in the same spectrum. Hence, the suggested isolated proton bands at 1203 and $1090 \mathrm{~cm}^{-1}$ correspond to the bands of deuterium modes at 882 (1.364) and $834(1.310)$ - in parenthesis we provide here the frequency isotopic ratios. In turn, the ND bands corresponding to the three bands of pure $\mathrm{NH}$ L-alanine at 544,500 and $415 \mathrm{~cm}^{-1}$ correspond to those appearing at 386 (1.395), 350 (1.430) and $312(1.330) \mathrm{cm}^{-1}$ in the far-IR spectra reported in [4]. Note that, taking into account their behavior upon deuteration, the bands at 544 and $415 \mathrm{~cm}^{-1}$ should be due to modes with a 


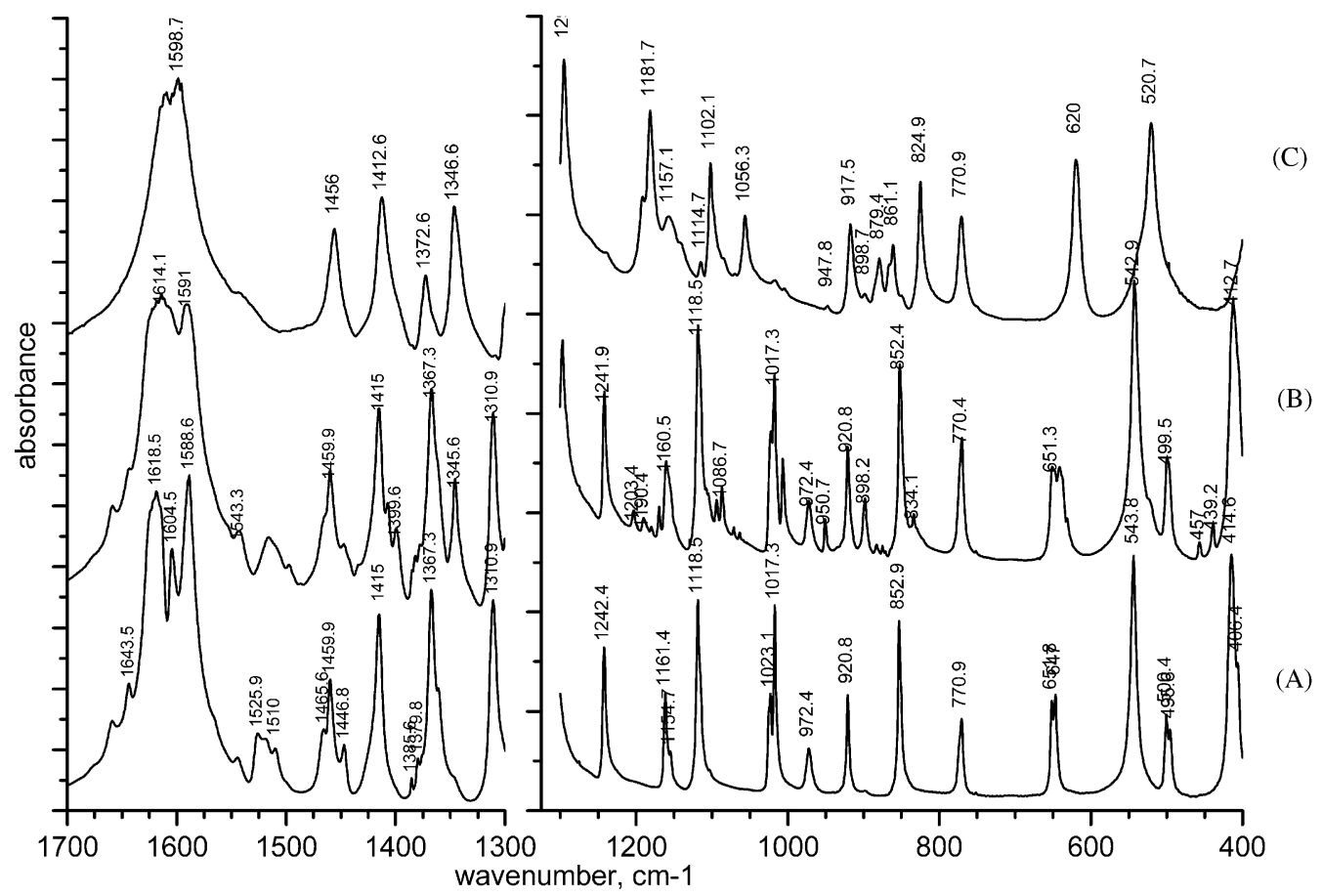

Fig. 5. The spectra of L-alanine at $10 \mathrm{~K}$ : (A) $100 \% \mathrm{NH}_{3}^{+}$, (B) $>90 \% \mathrm{NH}_{3}^{+}+\mathrm{NDH}_{2}^{+}$and (C) $>90 \% \mathrm{ND}_{3}^{+}+\mathrm{NHD}_{2}^{+}$.

predominant contribution from a $\mathrm{NH}$ proton coordinate. Hence, the previous assignments of these bands (see Table 2) are now revised. On the whole, nine relatively intense bands due to the $\mathrm{NH}_{3}^{+}$group of L-alanine could be identified in the spectral region below $2000 \mathrm{~cm}^{-1}$, all of them being sensitive to deuteration. All these bands have their $\mathrm{ND}_{3}^{+}$counterparts observable in the spectrum of the $\mathrm{ND}_{3}^{+}(>90 \%)$ L-alanine, showing isotopic ratios ranging from 1.32 to 1.43 (see Table 3).

The relatively small deviations of the frequency isotopic ratios found for these pairs of bands from the harmonic value clearly show that all modes are almost pure proton vibrations. In addition, since the frequencies of the bands of pure $\mathrm{NH}_{3}^{+}$group do not practically change when compared with those corresponding to the $\mathrm{NHD}_{2}^{+}$species, it can be concluded that the internal coupling of the different proton modes is small, as well as their coupling with skeletal coordinates. Note that this result contradicts the assumption, frequently found in the literature, which considers that proton bending modes in this type of compounds should have a significant degree of coupling with skeletal coordinates.

As mentioned above, our observations do not confirm the assignment of the band at ca. 1370 $\mathrm{cm}^{-1}$ to a $\mathrm{CH}_{3}$ bending mode [4]. In addition, the previous assignment of the band at $1310 \mathrm{~cm}^{-1}$ to a vibration of the $\mathrm{C}-\mathrm{H}$ group [2] does not also appear to be correct, since this band was found to be strongly affected by deuteration. The previous assignment of the band at $972 \mathrm{~cm}^{-1}$ to the overtone of the band observed at ca. $500 \mathrm{~cm}^{-1}$ [4] is also questionable, since the intensities of these bands are comparable, while it could be expected that the overtone appear as a relatively less intense feature.

It is interesting also to compare the spectra now obtained in the region below $2000 \mathrm{~cm}^{-1}$ with the one obtained previously for L-alanine isolated in the $\mathrm{KBr}$ matrix [17]. The bands observed in the spectrum of the $\mathrm{KBr}$ isolated substance at 1458 , 1397 and $769 \mathrm{~cm}^{-1}$ can be correlated with the bands now observed at 1460, 1415 and $770 \mathrm{~cm}^{-1}$, which are not affected by deuteration and, as 
Table 2

Peak frequencies, widths (in parentheses) and qualitative relative intensities of bands in pure and isotopically diluted L-alanine polycrystals at $10 \mathrm{~K}$ in the $2000-400 \mathrm{~cm}^{-1}$ range

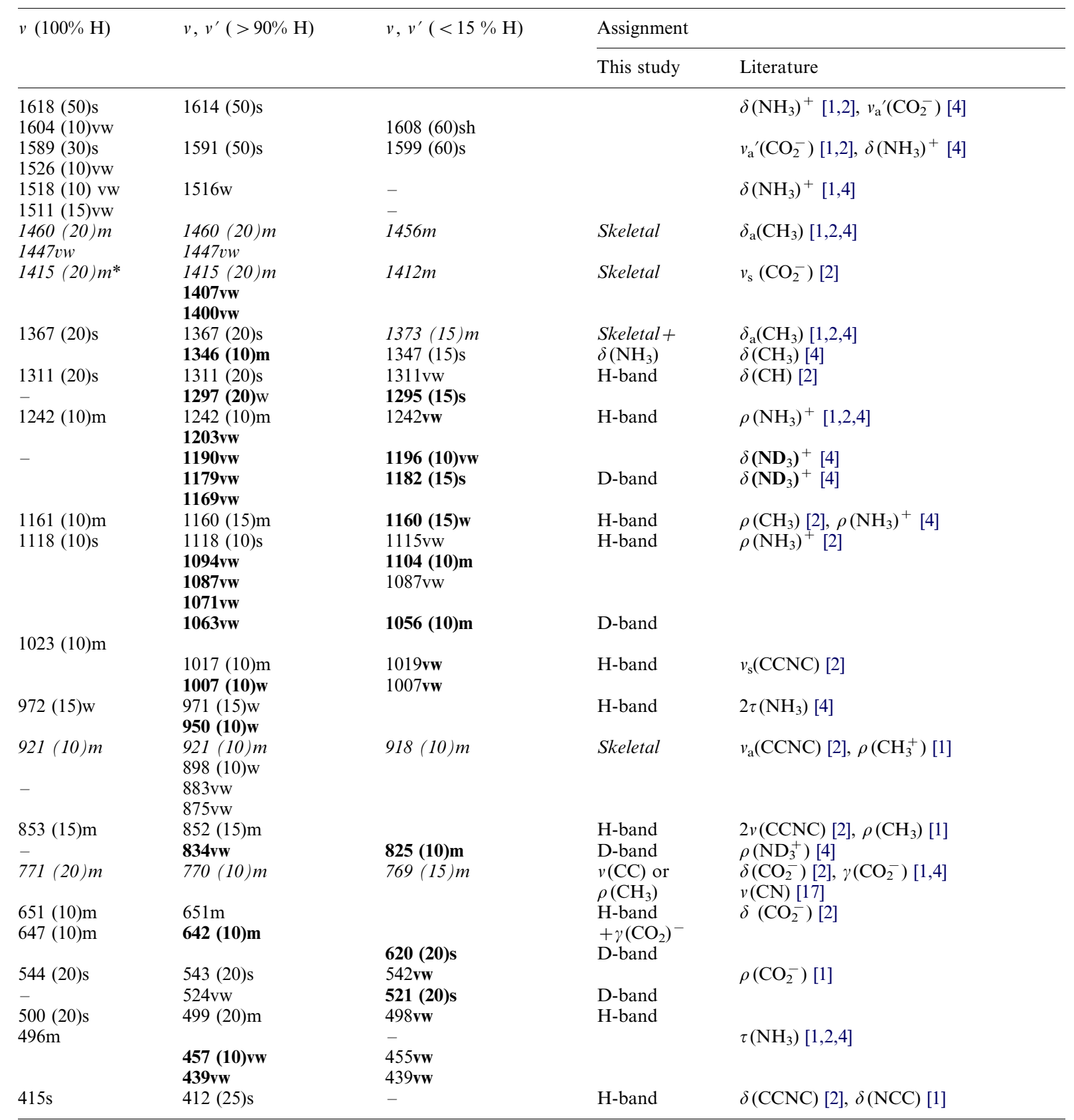

$v, \delta, \gamma, \tau$-stretching, bending, wagging and torsion modes, respectively. $\mathrm{s}, \mathrm{m}, \mathrm{w}, \mathrm{vw}$ - qualitative notation of the band intensities - strong, medium, weak and very weak, respectively. Italic - bands which are not affected with D-exchange and present in all three samples. Bold - new bands, which appear at ND doped sample relatively initial $(100 \% \mathrm{NH})$ sample. Bold vw - relate to the bands, which almost disappear in $\mathrm{ND}_{3}^{+}$sample relatively the ND doped sample. 
Table 3

The bands sensitive to D-exchange in the spectrum of $\mathrm{NH}_{\mathrm{L}-}$ alanine, their counterparts in D-L-alanine, isotopic ratios and relative integral intensities

\begin{tabular}{lrcl}
\hline $100 \% \mathrm{H}$ & Relative integral intensities, $\%$ & $<10 \% \mathrm{H}$ & $v / v^{\prime}$ \\
\hline 1618 & & 1181 & 1.370 \\
1526 & & 1102 & 1.385 \\
1367 & & 1056 & 1.290 \\
1242 & 4 & 870 & 1.427 \\
1118 & 12 & 825 & 1.355 \\
1017 & 8 & 771 & 1.320 \\
972 & 4 & - & - \\
853 & 8 & 620 & 1.376 \\
647 & 8 & - & - \\
543 & 16 & $386[4]$ & 1.410 \\
500 & 8 & $352[4]$ & 1.430 \\
414 & 32 & $312[4]$ & 1.330 \\
\hline
\end{tabular}

already mentioned before, are ascribable to skeletal or $\mathrm{CH}$ bending modes [17]. The assignment of the band at $769 \mathrm{~cm}^{-1}$ to the $\mathrm{CN}$ stretching mode [17] may be questioned, since this band does not change both on cooling and deuteration and does not also seem to be affected by $\mathrm{H}$-bond formation or strengthening (its position does not change on going from the $\mathrm{KBr}$ matrix to the crystalline state), while it should be somewhat affected by these changes. For example, deuteration of the $\mathrm{NH}_{3}^{+}$ group leads to a relative change of its mass of ca. $9 \%$, which should be reflected in a detectable shift of the band position to lower frequencies. This band can also be correlated with the band observed at $782 \mathrm{~cm}^{-1}$ in the low-temperature argon matrix spectrum of neutral L-alanine [18]. Hence, the position of this band is even relatively insensitive to the migration of the proton from the carboxylic towards the amino group and is much probably due to a $\mathrm{C}-\mathrm{C}$ stretching vibration or methyl rocking mode. The band observed in the $\mathrm{KBr}$ matrix isolated $\mathrm{L}$-alanine at $1358 \mathrm{~cm}^{-1}$, assigned to the $\mathrm{CO}_{2}^{-}$scissoring mode [17], should have its counterpart at $1372 \mathrm{~cm}^{-1}$ in the spectrum of the crystal. This latter band can only be clearly seen in the spectrum after deuteration, since the superimposed intensive band due to the $\mathrm{NH}$ bending mode observed at $1370 \mathrm{~cm}^{-1}$ then shifts to lower frequencies. Finally, the two strong bands appearing in the $\mathrm{KBr}$ matrix isolated zwitterion at
1121 and $855 \mathrm{~cm}^{-1}$ have also practically coincident counterpart bands in the crystalline phase spectrum of pure $\mathrm{NH}$ L-alanine (bands at 1118 and $853 \mathrm{~cm}^{-1}$ ). These bands disappear upon deuteration and then shall be proton-related bands. Hence, their previous assignments to $\mathrm{CH}_{3}$ bending and skeletal modes, respectively [17], were now revised.

The two components of the band at $650 \mathrm{~cm}^{-1}$ observed at $10 \mathrm{~K}$ in the spectrum of pure $\mathrm{NH} \mathrm{L}_{\mathrm{L}}$ alanine (Fig. 3C) reveal different behavior upon addition of $10 \%$ deuterium. The intensity of the band component at $652 \mathrm{~cm}^{-1}$ does not change, while the second component, at $647 \mathrm{~cm}^{-1}$, becomes more than two times weaker, and a new, relatively intensive band at $642 \mathrm{~cm}^{-1}$ appears in the spectrum (Fig. 4B). The decrease of intensity of the component at $647 \mathrm{~cm}^{-1}$ approximately correlates with the intensity of the new band. These features are with all probability due to the out-ofplane $\mathrm{CO}_{2}^{-}$mode $\left[\gamma\left(\mathrm{CO}_{2}^{-}\right)\right]$which can be expected to be extensively coupled with the $\mathrm{NH}(\mathrm{D}) v_{4}$ mode.

In the spectrum of $\mathrm{NH}$ L-alanine, there are essentially three bands which show a high sensitivity to the temperature change, all of them exhibiting a similar pattern of variation: blue shift, narrowing and splitting into two components on cooling. These bands deserve here further comments. The lowest frequency band was previously observed in the $\mathrm{NH}$ L-alanine far-IR spectrum $[3,4]$, appearing at $350 \mathrm{~cm}^{-1}$. On cooling, this band splits giving rise to two bands separated by ca. $20-25 \mathrm{~cm}^{-1}$, the gravity center of the bands shifting up by approximately $10 \mathrm{~cm}^{-1}$. The splitted bands have a bandwidth smaller than 3 $\mathrm{cm}^{-1}$. The second band is observed at ca. 500 $\mathrm{cm}^{-1}$. On cooling, it shifts upwards by ca. 10 $\mathrm{cm}^{-1}$ and splits into two bands (with bandwidths less than $5 \mathrm{~cm}^{-1}$ ) separated by $5 \mathrm{~cm}^{-1}$. The highest frequency band showing high sensitivity to the temperature variation is observed at $972 \mathrm{~cm}^{-1}$. It shows a blue shift of ca. $8 \mathrm{~cm}^{-1}$ and its bandwidth reduces from 18 to $9 \mathrm{~cm}^{-1}$. In this case, no splitting could be observed since this band is too wide, even at low temperature, to enable the expected splitting to be clearly noticed.

Bandekar et al. [3] have discussed previously the "striking" temperature-dependent behavior of the 
band at $350 \mathrm{~cm}^{-1}$, the splitting of which at cooling was assigned to the lifting of the accidental degeneracy of the $\mathrm{CCN}$ deformation skeletal mode (59\% PED) mixed with the $\mathrm{NH}_{3}$ torsion ( $21 \%$ PED). However, the temperature dependence shown by this band and by those observed at 500 and $972 \mathrm{~cm}^{-1}$ is typical for H-bondingrelated proton bands [12-14]. As mentioned before, the 500 and $972 \mathrm{~cm}^{-1}$ bands disappear upon deuteration (Fig. 4A and C) which is in consonance with their assignment to proton-related vibrations. In the case of the $350 \mathrm{~cm}^{-1}$ feature, more detailed far-IR data recently published [4], also unequivocally shows that both component bands resulting from low-temperature splitting are sensitive to deuteration. Accordingly, the assignment of this band shall also be changed.

The temperature splitting of all three bands now discussed (Fig. 3) indicates that in crystalline Lalanine there is a non-negligible disorder associated with the possibility the protons have to occupy different positions in the H-bonds established by the $\mathrm{NH}_{3}^{+}$group.

\section{Conclusion}

In summary, the following main conclusions can be drawn from this study:

i) The three decoupled $v_{1}$ bands observed in the spectra of $\mathrm{NHD}_{2}^{+}$L-alanine correspond to structure forming (ordered) $\mathrm{H}$-bonds in the Lalanine crystal, which were found by X-ray and neutron scattering methods. From their peak positions or their frequency red shifts relative to the reference frequency of a free $\mathrm{NH}$ stretching mode, the energies of the three different types of structure forming $\mathrm{H}$-bonds (or contributions of $\mathrm{H}$-bonds to the lattice energy) could be estimated using an empirical correlation. The obtained values were 21.5, 25 and $34 \mathrm{~kJ} \mathrm{~mol}^{-1}$, and their sum compare relatively well with the binding energy found experimentally for the L-alanine zwitterion dimer. Other NH stretching bands, corresponding to essentially non-ordered $\mathrm{NH}_{3}^{+}$ groups, cannot be resolved reliably as their intensities and widths depend on the frequency; the resulting bands are either narrow but too weak (for weak H-bonds) or intense but too broad (for strong H-bonds).

ii) Comparison of the temperature and isotopic behavior of the bands in the spectra of all four isotopomers of L-alanine studied $\mathrm{NH}_{3}^{+}$, $\mathrm{NDH}_{2}^{+}, \mathrm{NHD}_{2}^{+}$and $\mathrm{ND}_{3}^{+}$), enabled to establish reliable assignments for all nine sensitive to isotope exchange bands of pure $\mathrm{NH} \mathrm{L-}$ alanine appearing below $1250 \mathrm{~cm}^{-1}$ to deformational (presumably $v_{4}$ ) modes of protons, which are differently $\mathrm{H}$-bonded in the crystal. The appearance of several (at least eight) new proton bands in the spectrum of the $\mathrm{NDH}_{2}^{+} \mathrm{L}_{-}$ alanine molecule in the doped $(<10 \% \mathrm{D}) \mathrm{L}$ alanine crystal indicates the existence of different accessible proton positions in $\mathrm{H}$-bonds within the crystal, which also can be described as an intrinsic $\mathrm{H}$-bond disorder, leading also to observation of temperature induced band splitting. Observation in the spectra of the crystal of NH bands, appearing at frequencies identical to those observed for $\mathrm{L}$-alanine zwitterions isolated in $\mathrm{KBr}$ matrix (e.g. the bands appearing at 1115 and $\left.851 \mathrm{~cm}^{-1}\right)$, strongly suggests the presence of relatively weakly bound $\mathrm{NH}_{3}^{+}$protons in the crystal, in addition to the structurally more fixed $\mathrm{H}$ bonding groups.

iii) In contrast to what occurs in the $\mathrm{NH}$ stretching spectral region, all kinds of $\mathrm{NH}_{3}^{+}$groups are clearly observed in the out-of-plane mode spectral region. These bands are rather narrow and well resolved, especially in isotopically doped cold crystals. Their peak positions depend mainly on the H-bond strength but their intensity is frequency-independent and seems to reflect the relative abundance of each $\mathrm{H}$-bond. As a whole, the results indicate that in the L-alanine crystal more types of H-bonds are present than are revealed by structural methods. The most intensive bands appearing in the low-frequency spectral region, at 415 and $544 \mathrm{~cm}^{-1}$, with an intensity ratio $2: 1$ can be assigned to the $v_{4}$ bands of the structurally more ordered $\mathrm{NH}_{3}^{+}$groups and correlated with the $v_{1}$ proton bands corresponding to 
three H-bond types existing in the crystal with energies 21.5, 25 and $34 \mathrm{~kJ} \mathrm{~mol}^{-1}$, which are directly accessible to structural methods (Xray, neutron diffraction). From the integral intensities of the $v_{4}$ bands, the relative abundance of each type of $\mathrm{H}$-bond can be estimated: the ordered H-bonds correspond approximately to $48 \%$ of all $\mathrm{H}$-bonds in the crystal (Table 3); more than $30 \%$ of protons form stronger $\mathrm{H}$-bonds, giving rise to $\mathrm{NH}$ deformation modes at higher frequencies; approximately $20 \%$ protons are weakly bound in the crystal (absorbing at frequencies close to those observed in L-alanine zwitterions isolated in a $\mathrm{KBr}$ matrix [17]). The larger number of H-bond contacts found by infrared spectroscopy as compared with structural methods may be due to the sensitivity of the former to short-range interactions as compared with the long-range periodic order seen by the latter.

iv) Mentioned in [4] as "anomalous", the temperature dependence of the bands observed at 350 [4], 500, 972 and $1160 \mathrm{~cm}^{-1}$ is very similar to that exhibited by the out-of-plane bending IR bands of other $\mathrm{H}$-bonded molecules in crystalline phase, such as alcohols $[13,14]$ and carbohydrates [5]. In all such systems, these bands show directional shift of maximum to higher frequencies and bandwidth narrowing on cooling, which correlates with the freezing of the hot transitions from low-frequency intermolecular deformational modes and the concomitant strengthening of the $\mathrm{H}$-bonds (this phenomenon was described and generalized in [12]).

This study shows also that the proton vibrational modes of L-alanine can be interpreted using the terminology which is currently used in the description of vibrational spectra of simpler $\mathrm{H}$ bonded systems, which do not include the pyramidal $\mathrm{NH}_{3}^{+}$group. Usually, when the vibrational modes of $\mathrm{NH}_{2}$ or $\mathrm{NH}_{3}^{+}$groups are discussed, the stretching modes (for symmetry reasons) are described as antisymmetric or symmetric and the deformation vibrations as wagging, scissoring, rocking and twisting modes in isolated molecules; this local symmetry adapted normal mode descrip- tion implies the simultaneous movement of the locally equivalent-by-symmetry protons. In the case of crystalline L-alanine, however, the local symmetry of $\mathrm{NH}_{3}^{+}$ion is broken due to multiple heterogeneous $\mathrm{H}$-bonding and the vibrations of each proton become essentially non-coupled. In cases like this, the assignment of the vibrational spectra on the basis of individual $\mathrm{H}$-bonded $\mathrm{NH}$ moieties seems to be more reasonable. Hence, for such systems, the vibrational H-bonding properties of $\mathrm{NH}_{2}$ or $\mathrm{NH}_{3}^{+}$groups can be defined in a simpler way, which enables a direct comparison with those of the simpler intermolecular H-complexes of $\mathrm{OH}$ and $\mathrm{NH}$ groups.

\section{Acknowledgements}

M.R. gratefully acknowledges financial support from the Israel Ministry for Immigrant Absorption.

\section{References}

[1] H. Susi, D.M. Byler, J. Mol. Struct. 63 (1980) 1.

[2] C.H. Wang, R.D. Storms, J. Chem. Phys. 55 (1971) 3291.

[3] J. Bandekar, L. Genzel, F. Kremer, L. Santo, Spectrochim. Acta A 39 (1983) 357.

[4] (a) M. Barthes, A.F. Vik, A. Spire, H.N. Bordallo, J. Eckert, J. Phys. Chem. A 106 (2002) 5230;

(b) H.N. Bordallo, M. Barthes, J. Eckert, Physica B $241-$ 243 (1998) 1138.

[5] M. Rozenberg, A. Loewenschuss, Y. Marcus, Carbohydr. Res. 328 (2000) 307.

[6] M. Rozenberg, A.V. Iogansen, A.A. Mashkovsky, S.E. Odinokov, Spectrosc. Lett. 5 (1972) 75.

[7] J. Bandekar, G. Zundel, Spectrochim. Acta A 38 (1982) 815.

[8] G. Zerbi, G. Dellepiane, J. Raman Spectrosc. 12 (1982) 165.

[9] S.C.S. Bugalho, E.M.S. Maçôoas, M.L.S. Cristiano e, R. Fausto, PCCP 3 (2001) 3541.

[10] M.S. Lehmann, T.F. Koetzle, W.C. Hamilton, J. Am. Chem. Soc. 94 (1972) 2657.

[11] R. Destro, R.E. Marsh, R. Bianchi, J. Phys. Chem. 92 (1988) 966.

[12] A.V. Iogansen, Izvestiya Akademii Nauk SSSR, Seriya Fizicheskaya 53 (1989) 1741 [Engl. Transl. 1989 by Allerton Press, Inc.].

[13] M. Rozenberg, A.V. Iogansen, Opt. Spectrosc. (USSR) 64 (1988) 693. 
[14] A.V. Iogansen, M. Rozenberg, J. Struct. Chem. (USSR) 30 (1989) 76.

[15] A.V. Iogansen, Spectrochim. Acta A 55 (1999) 1585.

[16] M. Rozenberg, A. Loewenschuss, H.-D. Lutz, Y. Marcus, Carbohydr. Res. 315 (1999) 89.

[17] X. Cao, G. Fisher, Chem. Phys. 255 (2000) 195.

[18] S.G. Stepanian, I.D. Reva, E.D. Radchenko, L. Adamowicz, J. Phys. Chem. A 102 (1998) 4623.
[19] M. Rozenberg, A. Loewenschuss, Y. Marcus, PCCP 2 (2000) 2699.

[20] R.K. Khanna, M. Horak, E.R. Lippincott, Spectrochim. Acta 22 (1966) 1759.

[21] P.-G. Jonsson, A. Kvick, Acta Cryst. B 28 (1972) 1827.

[22] W.D. Price, P.D. Schnier, E.R. Williams, J. Phys. Chem. B 101 (1997) 664. 